\title{
Effects of antenatal magnesium sulfate treatment for neonatal neuro-protection on cerebral oxygen kinetics
}

\author{
Michael J. Stark ${ }^{1,2}$, Nicolette A. Hodyl' and Chad C. Andersen ${ }^{1,2}$
}

BACKGROUND: The underlying neuro-protective mechanisms of antenatal magnesium sulfate $\left(\mathrm{MgSO}_{4}\right)$ in infants born preterm remain poorly understood. Early neonatal brain injury may be preceded by low cerebral blood flow (CBF) and elevated cerebral fractional tissue oxygen extraction (CFTOE). This study investigated the effect of antenatal $\mathrm{MgSO}_{4}$ on cerebral oxygen delivery, consumption, and cFTOE in preterm infants.

METHODS: CBF and tissue oxygenation index were measured, and oxygen delivery, consumption, and CFTOE calculated within $24 \mathrm{~h}$ of birth and at 48 and $72 \mathrm{~h}$ of life in 36 infants $\leq 30 \mathrm{wk}$ gestation exposed to $\mathrm{MgSO}_{4}$ and 29 unexposed infants.

RESULTS: Total internal carotid blood flow and cerebral oxygen delivery did not differ between the groups at the three study time-points. Cerebral oxygen consumption and cFTOE were lower in infants exposed to antenatal $\mathrm{MgSO}_{4}(P=0.012)$ compared to unexposed infants within $24 \mathrm{~h}$ of delivery. This difference was not evident by $48 \mathrm{~h}$ of age. Fewer infants in the $\mathrm{MgSO}_{4}$ group developed $\mathrm{P} / \mathrm{IVH}$ by $72 \mathrm{~h}$ of age $(P=0.03)$.

CONCLUSION: Infants exposed to $\mathrm{MgSO}_{4}$ had similar systemic and cerebral hemodynamics but lower CFTOE compared to nonexposed. These findings suggest reduced cerebral metabolism maybe a component of the neuro-protective actions of antenatal $\mathrm{MgSO}_{4}$.

D espite significant improvements in survival for infants born preterm, the rate of major neurodevelopmental impairment in survivors has not diminished (1). As a result, strategies designed to reduce adverse neurological outcomes have been a major perinatal research focus. Antenatal magnesium sulfate $\left(\mathrm{MgSO}_{4}\right)$ is one such strategy. Following the first report of an association between perinatal administration of $\mathrm{MgSO}_{4}$ and a reduction in the risk of peri/intraventricular hemorrhage $(\mathrm{P} / \mathrm{IVH})(2)$, its use as a neuro-protective therapy when given to women at risk of preterm birth has become established practice (3). While $\mathrm{MgSO}_{4}$ has been shown to decrease the risk of P/IVH (4), cerebral palsy and the rate of substantial gross motor dysfunction (5), the mechanisms underlying these effects remain poorly understood.

Proposed mechanisms by which $\mathrm{MgSO}_{4}$ exerts a neuroprotective effect include effects on the cardiovascular system through its role in the regulation of vascular tone (6), cerebral metabolism including prevention of excess glutamate release (7), and reductions in systemic proinflammatory cytokine production (8). The effect of antenatal $\mathrm{MgSO}_{4}$ administration on the neonatal systemic and cerebral vasculature is unclear. While increased cerebral blood flow (CBF) velocities have been reported in infants whose mothers received $\mathrm{MgSO}_{4}$ for the management of pre-eclampsia or tocolysis $(9,10)$ another study has reported significant lowering of neonatal cerebral perfusion (11). The sole study reporting neonatal cardiovascular effects of antenatal $\mathrm{MgSO}_{4}$ for neuro-protection found no difference in echocardiographic measures of systemic blood flow on the first day of life (12).

For extremely preterm infants, the transition to extrauterine life is characterized by low baseline $\mathrm{CBF}$ and high cerebral oxygen consumption (13), with cerebral oxygen consumption reflecting cerebral metabolic activity (14). Elevated cerebral fractional tissue oxygen extraction (cFTOE), derived by near infrared spectroscopy (NIRS), is a readily measurable variable of the relationship between cerebral oxygen delivery and consumption and precedes early neonatal brain injury following very preterm birth $(15,16)$. While studies in fetal sheep have reported temporary reductions in cerebral oxygen consumption following $\mathrm{MgSO}_{4}$ administration (17), it is only recently that similar effects have been described in infants born to women given antenatal $\mathrm{MgSO}_{4}$ for pre-eclampsia (18). We hypothesized that reduced neonatal cerebral oxygen consumption contributes to the observed neuro-protective actions of antenatally administered $\mathrm{MgSO}_{4}$. Therefore, the aim of the current study was to investigate the effect of antenatal $\mathrm{MgSO}_{4}$ on cerebral oxygen delivery, consumption and cFTOE in preterm infants less than $30 \mathrm{wk}$ gestation.

\section{RESULTS}

Clinical characteristics of infants are shown in Table 1. Thirtysix infants were exposed to $\mathrm{MgSO}_{4}$ for neuro-protection, with three receiving a $4 \mathrm{~g}$ loading dose alone prior to preterm delivery. The mean (minimum-maximum) maintenance dose received was $8 \mathrm{~g}(0.5-23 \mathrm{~g})$. There was no significant difference for any clinical characteristics between those infants exposed to $\mathrm{MgSO}_{4}$ prior to delivery and those not. For the 28 infants 
not exposed to antenatal $\mathrm{MgSO}_{4}, 12$ (43\%) delivered vaginally within $1 \mathrm{~h}$ of presentation to hospital and $16(57 \%)$ required urgent emergency caesarean section following presentation (seven as a result of a significant cardiotocographic abnormality (such as persistent fetal tachycardia or prolonged fetal heart rate decelerations), six secondary to antepartum hemorrhage, two secondary to acute maternal sepsis/chorioamnionitis, and one following umbilical cord prolapse). The mean (SD) age of the infants at time of the initial NIRS and cranial ultrasound study was 11 (6) hours for the nonexposed group and 12 (7) hours for the $\mathrm{MgSO}_{4}$ group. While four infants had cranial ultrasound evidence of P/IVH at the time of the NIRS study (all unilateral grade $1 \mathrm{IVH}$ ), fewer infants in the $\mathrm{MgSO}_{4}$ group $(n=4)$ were diagnosed with $\mathrm{P} / \mathrm{IVH}$ (all grades) compared to those not exposed to $\mathrm{MgSO}_{4}(n=9)$ by $72 \mathrm{~h}$ of life $(P=0.03)$.

Table 1. Clinical characteristics according to exposure to antenatal $\mathrm{MgSO}_{4}$

\begin{tabular}{lccc}
\hline & $\begin{array}{c}\text { No antenatal } \\
\mathrm{MgSO}_{4}(n=28)\end{array}$ & $\begin{array}{c}\text { Antenatal } \\
\mathrm{MgSO}_{4}(n=36)\end{array}$ & $P$ \\
\hline Gestational age (days) & $188(12)$ & $190(11)$ & 0.52 \\
Male & $18(64)$ & $16(45)$ & 0.07 \\
Birth weight centile & $41(34)$ & $33(35)$ & 0.17 \\
Histological chorioamnionitis & $11(39)$ & $14(39)$ & 0.60 \\
Completed antenatal steroids & $16(57)$ & $19(53)$ & 0.46 \\
Spontaneous vaginal delivery & $12(43)$ & $14(39)$ & 0.47 \\
Cord arterial pH & $7.25(0.15)$ & $7.26(0.05)$ & 0.80 \\
Cord arterial lactate (mmol/l) & $2.9(1.2)$ & $3.4(1.6)$ & 0.20 \\
5-min APGAR & $7(1)$ & $8(1)$ & 0.27 \\
Mechanical ventilation & $13(46)$ & $12(31)$ & 0.15 \\
Inotropic support & $10(36)$ & $15(42)$ & 0.12 \\
P/IVH > grade II & $9(32)$ & $4(11)$ & 0.03 \\
PDA & $15(54)$ & $11(31)$ & 0.06 \\
Hemoglobin at first study (g/l) & $141(22)$ & $143(21)$ & 0.53 \\
pCO at first study (mmHg) & $43(8)$ & $42(10)$ & 0.37 \\
SaO at first study (\%) & $95(4)$ & $94(4)$ & 0.2
\end{tabular}

Data are presented as median (SD) or number (\%). The presence of a significant patent ductus arteriosus (PDA) was defined as echocardiographic measurement of ductal diameter $>1.4 \mathrm{~mm}$ with bi-directional or left to right shunt.

$\mathrm{P} / \mathrm{IVH}$, peri/intraventricular hemorrhage.
A significant main effect for time was observed for mean arterial blood pressure (Figure 1a, $P=0.01$ ) and right ventricular output (Figure $1 \mathrm{~b}, P=0.02$ ). On post-hoc analysis mean arterial pressure at $72 \mathrm{~h}$ of age was significantly higher compared to $24 \mathrm{~h}(P<0.01)$ and $48 \mathrm{~h}(P=0.01)$ and right ventricular output significantly higher at $72 \mathrm{~h}$ of age compared to $24 \mathrm{~h}$ of age $(P<0.01)$. No significant time effect was seen for total internal carotid blood flow (Figure 1c, $P=0.19$ ). No effect of $\mathrm{MgSO}_{4}$ exposure was observed for any of these systemic hemodynamic variables at any time point. As a result, NIRS-derived modified cerebral oxygen delivery was similar across the study period (Figure 2a, $P=0.25$ ) and between the $\mathrm{MgSO}_{4}$ exposure groups.

A significant interaction effect for time and $\mathrm{MgSO}_{4}$ exposure was observed for modified cerebral oxygen consumption (Figure $2 \mathbf{b}, P=0.04$ ). On post-hoc analysis modified cerebral oxygen consumption was significantly lower within $24 \mathrm{~h}$ of age in those infants exposed to $\mathrm{MgSO}_{4}$ antenatally compared to the nonexposed group $(P=0.01)$. No significant difference between the groups was seen at either 48 or $72 \mathrm{~h}$ of age. A similar interaction effect between postnatal age and $\mathrm{MgSO}_{4}$ was seen for cFTOE (Figure 2c, $P=0.03$ ). On post-hoc analysis, cFTOE was significantly lower in those infants exposed to antenatal $\mathrm{MgSO}_{4}$ within $24 \mathrm{~h}$ of delivery $(P=0.01)$. This difference between the groups was no longer evident by $48 \mathrm{~h}$ of age (Figure 2b). Further, in the nonexposed group, cFTOE was significantly lower on day $2(P<0.01)$ and day $3(P=0.01)$ compared to day 1 of life, a temporal change that was not observed in the $\mathrm{MgSO}_{4}$ exposed infants. Regression modeling indicated no significant dose-dependent relationship between total magnesium sulfate dose and any systemic or cerebral hemodynamic variable.

\section{DISCUSSION}

In the current study, preterm infants $<30 \mathrm{wk}$ gestation exposed to antenatal $\mathrm{MgSO}_{4}$ for neuro-protection had significantly lower cFTOE than those not exposed to antenatal $\mathrm{MgSO}_{4}$ but similar measures of systemic and cerebral hemodynamics including mean blood pressure, right ventricular output and total internal carotid blood flow. Interestingly, this difference was confined to the first $24 \mathrm{~h}$ of life. With tissue oxygen extraction a dynamic variable primarily determined by oxygen
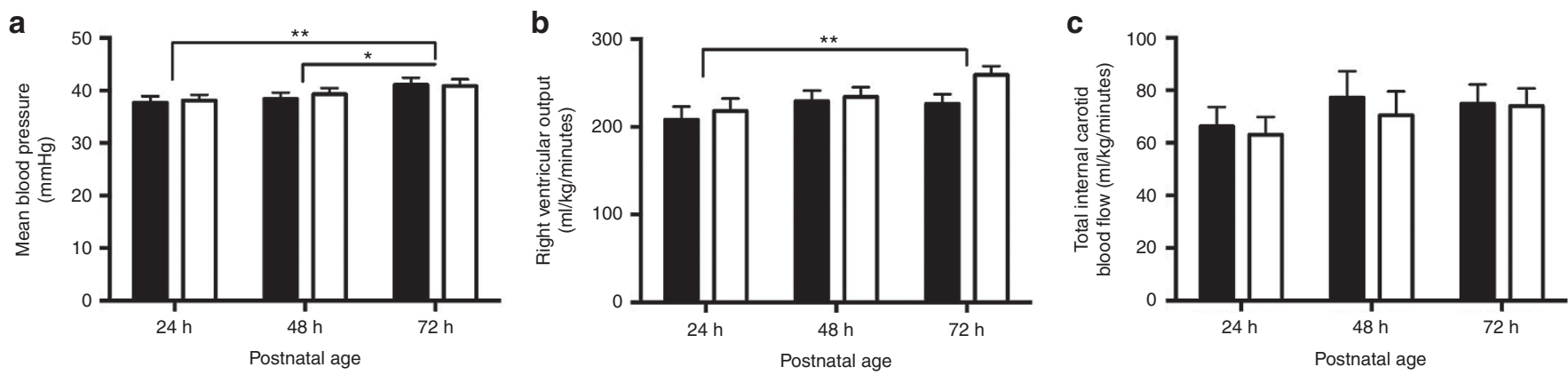

Figure 1. Postnatal alterations in systemic hemodynamics. (a) Invasive mean arterial blood pressure (mmHg), (b) right ventricular output (ml/kg/min), and (c) total internal carotid blood flow ( $\mathrm{ml} / \mathrm{kg} / \mathrm{min}$ ) in infants on days 1-3 of postnatal life. Black bars represent infants not exposed to $\mathrm{MgSO}_{4}$ and open bars represent infants exposed to antenatal $\mathrm{MgSO}_{4}$. For Panels $\mathbf{a}$ and $\mathbf{b},{ }^{*} P=0.01 ;{ }^{* *} P<0.01$. 


\section{Articles | Stark etal.}
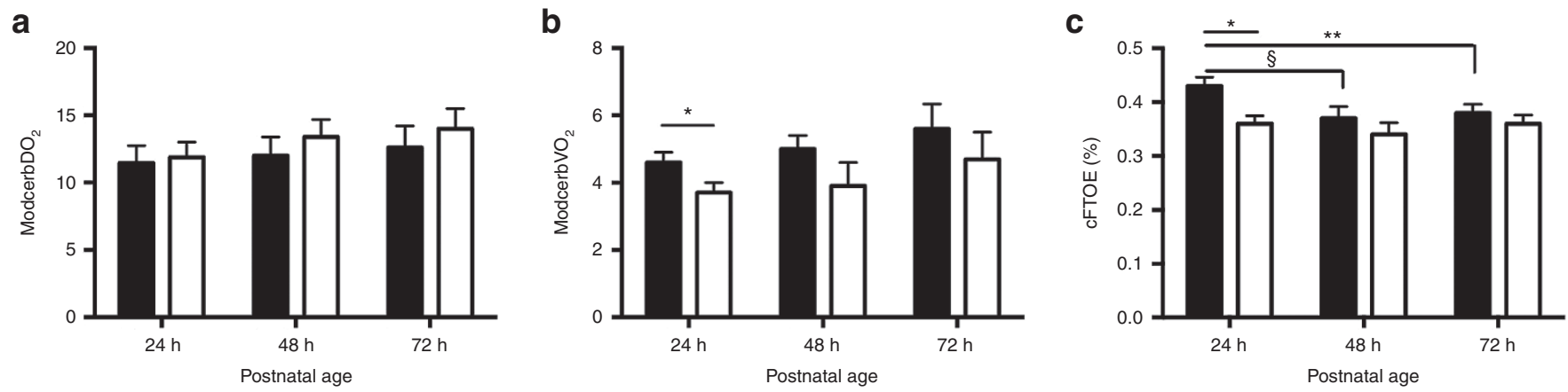

Figure 2. Postnatal changes in NIRS derived cerebral oxygen kinetics. (a) Modified cerebral oxygen delivery (ModcerbDO ${ }_{2}$ ), (b) modified cerebral oxygen consumption (ModcerbVO $)_{2}$, and (c) cerebral fractional tissue oxygen extraction (\%) in infants on days 1-3 of postnatal life. Black bars represent infants not exposed to $\mathrm{MgSO}_{4}$ and open bars represent infants exposed to antenatal $\mathrm{MgSO}_{4}$. For panel $\mathbf{b},{ }^{*} P=0.01$. For panel $\mathbf{c}$, ${ }^{*} P<0.05$, ${ }^{* *} P=0.01,{ }^{\S} P<0.01$.

consumption in the context of prevailing oxygen delivery, this data would suggest that one mechanism of action for $\mathrm{MgSO}_{4}$ in the very preterm newborn is a reduction in cerebral metabolic demand and therefore cFTOE. As elevated cFTOE in the immediate newborn period is predictive of early neonatal brain injury (15), the alteration in the balance of cerebral oxygen delivery and consumption resulting in reduced cFTOE may contribute to the neuro-protective actions of $\mathrm{MgSO}_{4}$. However, the lack of a linear relationship between any of the systemic and cerebral hemodynamic variables and $\mathrm{MgSO}_{4}$ dose received suggests a higher cumulative dose may not provide a greater degree of neuro-protection.

The majority of clinical studies investigating neonatal systemic and cerebral hemodynamic effects of $\mathrm{MgSO}_{4}$ have been in the setting of $\mathrm{MgSO}_{4}$ used as an antihypertensive for pregnancy-induced hypertension or as a tocolytic for threatened preterm labor $(9,11,19)$. These studies have reported conflicting results, with reduced (11), unchanged (19), and increased $\mathrm{CBF}$ velocities and cerebral vascular resistance in the postnatal period (9). The discrepancy in these findings may relate differences in gestational age of preterm infants investigated and the dose of $\mathrm{MgSO}_{4}$ administered. For instance, the loading (9) and continuous infusion dose (19) of antenatal $\mathrm{MgSO}_{4}$ for tocolysis is significantly higher than that used for neuro-protection in the current study. With elevated neonatal circulating magnesium levels known to persist beyond the initial transition to postnatal life (20), exposure to a lower total $\mathrm{MgSO}_{4}$ dose antenatally, with subsequent differences in neonatal plasma magnesium levels in the first few days of life, may also explain our observation that the difference in cFTOE was confined to the first $24 \mathrm{~h}$ of postnatal life.

While magnesium results in vasodilatation and reduced vascular reactivity (21), our data are consistent with the sole study of cardiovascular function following $\mathrm{MgSO}_{4}$ use for neuroprotection in infants of a comparable gestational age (12), with no differences in right ventricular output or mean arterial blood pressure observed. Further, we observed no differences in total internal carotid blood flow between the $\mathrm{MgSO}_{4}$ exposed and nonexposed infants, suggesting this therapy does not impact upon $\mathrm{CBF}$ and oxygen delivery.

There is little data focusing on alterations in cerebral oxygen consumption following antenatal $\mathrm{MgSO} 4$ exposure in preterm infants. The current study is, however, supported by animal studies, where a temporary reduction in cerebral oxygen consumption not associated with any alteration in CBF has been described in fetal sheep (17). Studies of preterm infants born to mothers with pregnancy-induced hypertension treated with antihypertensive medications, including $\mathrm{MgSO}_{4}$, have also reported reduced cFTOE in the first $24 \mathrm{~h}$ of life (18). The generalization of this latter study to preterm infants of normotensive mothers, however, is limited, given that pregnancy induced hypertension results in alterations in placental blood flow (22), and both fetal and neonatal circulations $(23,24)$. For instance, CBF may be greater in infants with evidence of brain-sparing in pregnancies complicated by pregnancy induced hypertension as a result of changes in fetal cerebral vascular resistance (23). To avoid the potential confounding effects of maternal hypertension in pregnancy on neonatal cerebral oxygen delivery and consumption, we excluded mothers who received any hypertensive medications, including $\mathrm{MgSO}_{4}$. However, our observation of a significantly lower cFTOE in those preterm infants exposed to $\mathrm{MgSO}_{4}$ purely for neuro-protection is consistent with this previous report (18), and supports MgSO4induced changes to cerebral oxygen kinetics.

While focusing on cerebral oxygen kinetics following exposure to antenatal $\mathrm{MgSO}_{4}$, this study did not aim to characterize the mechanisms through which cerebral oxygen consumption and therefore extraction was altered. It is known that $\mathrm{MgSO}_{4}$ rapidly crosses the placenta (25) and fetal blood brain barrier (26) blocking n-methyl-d-aspartate receptors (27) and glutamate receptors (28), receptors critically involved in neuronal death during hypoxic-ischemic injury. Whether these actions result in reduced neuronal excitation remains open to question (29). Recent evidence also supports a role for $\mathrm{MgSO}_{4}$ in immunomodulation with in vitro exposure of neonatal monocytes to clinically relevant doses of $\mathrm{MgSO}_{4}$ producing a reduction in both constitutive and toll-like receptor-stimulated TNF $\alpha$ and IL-6 gene and protein expression (8). Animal data indicate that neonatal cerebral oxygen consumption is elevated following exposure to in utero inflammation (30). It is therefore possible that protection from inflammatory processes conferred by $\mathrm{MgSO}_{4}$ could manifest as reduced cerebral oxygen consumption and cFTOE. However, further investigation is required of the mechanisms of $\mathrm{MgSO}_{4}$ action, particularly in light of a 
recent long-term follow-up study that reported no significant neuro-developmental benefit at school age of antenatal $\mathrm{MgSO}_{4}$ exposure (31).

There are limitations to the current study. With antenatal $\mathrm{MgSO}_{4}$ a proven antenatal therapy for neuro-protection (3), we could not randomize women presenting in preterm labor to receive $\mathrm{MgSO}_{4}$ or not. While those in the nonexposed group did not receive antenatal $\mathrm{MgSO}_{4}$ as a result of rapid delivery following presentation, there was no difference between the groups for the underlying etiology of their preterm delivery or the physiological stability of the infants following birth. With respect to the determination of cerebral oxygen delivery and consumption, the NIRS data was derived from a single sensor and CBF inferred from total internal carotid blood flow. However, NIRSderived cFTOE varies minimally between regions of the brain (32) and the current data are consistent with frequency domain NIRS measurements from multiple regions of the brain (33). Further, episodic sampling of dynamic measures, such as total internal carotid blood flow, may result in over-interpretation, particularly, if the duration and severity of adverse oxygen handling varies between infants. However, measurement of total internal carotid flow does provide greater insight into cerebral hemodynamics than measures of maximal or mean cerebral artery velocities commonly reported previously. Due to the in vivo nature of the current study, it was impossible to investigate the underlying mechanistic pathways relating antenatal $\mathrm{MgSO}_{4}$ to reduced cerebral oxygen consumption and cFTOE. Further, while this study identified a lower incidence of $\mathrm{P} / \mathrm{IVH}$ in the $\mathrm{MgSO}_{4}$-exposed group, we acknowledge that the study was not designed to assess this outcome and is there for underpowered to adequately address this question. Nonetheless this finding is consistent with previous reports of reduced rates of $\mathrm{P} /$ IVH following antenatal $\mathrm{MgSO}_{4}$ exposure (4).

In summary, antenatal exposure to $\mathrm{MgSO}_{4}$ for neuroprotection was associated with reduced cerebral oxygen consumption and cFTOE in very preterm infants without alterations in systemic or cerebral hemodynamics. While maintenance of adequate oxygen delivery while avoiding restricted oxygen consumption is critically important in the preterm newborn, this data highlights the importance of considering both sides of the oxygen delivery-consumption relationship in understanding antecedents to acquired brain injury. While the exact mechanism/s through which $\mathrm{MgSO}_{4}$ exerts a neuroprotective effect remain unknown, the current data suggest that reduced cerebral metabolic load may contribute to this beneficial action.

\section{METHODS}

\section{Study Participants}

In this observational study, preterm newborns $\leq 30 \mathrm{wk}$ gestational age, admitted to the neonatal intensive care unit of the Women's and Children's Hospital, Adelaide were recruited. Exclusion criteria included maternal use of antihypertensive medications, including $\mathrm{MgSO}_{4}$, for essential hypertension or pregnancy-induced hypertension, and those infants with life threatening congenital abnormalities or congenital heart disease. Clinical characteristics were recorded from the medical records. Antenatal exposure to $\mathrm{MgSO}_{4}$ for neuroprotection was defined as maternal administration of a $4 \mathrm{~g} \mathrm{MgSO}_{4}$ loading dose $\pm 1 \mathrm{~g} \mathrm{MgSO}_{4}$ per hour for a maximum of $24 \mathrm{~h}$ prior to delivery (34). No women received multiple courses of $\mathrm{MgSO}_{4}$. The presence of a significant patent ductus arteriosus was defined as echocardiographic measurement of ductal diameter $>1.4 \mathrm{~mm}$ with bidirectional or left to right shunt. Cranial ultrasounds were performed on days 1 and 3 of life with P/IVH defined as per Papile (35). All infants received a $20 \mathrm{mg} / \mathrm{kg}$-loading dose of intravenous caffeine on day 1 of life prior to a daily $10 \mathrm{mg} / \mathrm{kg}$ daily maintenance dose. The institutional human research ethics committee approved the study and parental consent was obtained for each newborn prior to inclusion.

\section{Cerebral Oxygen Delivery and Consumption}

Oxygen saturation was maintained within a target band $(85-95 \%)$ and $\mathrm{pCO}_{2}$ within the range of $45-55 \mathrm{mmHg}$. NIRS and cranial ultrasound studies were performed within $24 \mathrm{~h}$ of birth and repeated at 48 and $72 \mathrm{~h}$ of life. NIRS and cerebral ultrasound were not performed within $2 \mathrm{~h}$ of clinical interventions that are known to alter cerebral oxygen supply and extraction, including administration of surfactant (36), indomethacin/ibuprofen (37), and caffeine (38).

Tissue oxygen index (TOI) and systemic arterial saturation $\left(\mathrm{SaO}_{2}\right)$ were measured by NIRS (Hamamatsu NIRO-200, Hamamatsu Photonics K. K Hamamatsu City, Japan) and co-oximetry as described previously (15). Briefly, the sensor was placed on the right fronto-temporal region and NIRS data and contemporaneous $\mathrm{SaO}_{2}$ was captured at 1 s intervals. A 10 -min epoch of stable NIRS and $\mathrm{SaO}_{2}^{2}$ data (from a 30 -min recording period) were averaged with TOI, as a surrogate for cerebral venous oxygen saturation (15), and $\mathrm{SaO}_{2}$ values used for the oxygen kinetic equations.

At the end of each NIRS study, internal carotid artery blood flow measured by pulsed-wave Doppler ultrasound measurement of the internal carotid artery using an $8 \mathrm{MHz}$ linear phased-array transducer (Philips iE33 Ultrasound System, Andover, MA) (15) and right and left ventricular output and patency of the ductus arteriosus (including flow direction) were determined by functional echocardiography. $\mathrm{An}$ arterial hemoglobin concentration $[\mathrm{Hb}]$, lactate, $\mathrm{pH}, \mathrm{paO}_{2}$, and $\mathrm{paCO}_{2}$ were measured by co-oximeter (128 wavelength, spectrophotometer Radiometer Copenhagen, ABL 725, Denmark).

\section{Calculations}

cFTOE was calculated from the formula: $\mathrm{cFTOE}=\left[\left(\left(\left(\mathrm{SaO}_{2}-\mathrm{TOI}\right) /\right.\right.\right.$ $\mathrm{SaO}_{2}$ ) where $\mathrm{SaO}_{2}=$ systemic arterial saturation (co-oximetry) and TOI was used in place of cerebral venous oxygen saturation (15). Modified cerebral oxygen delivery $\left(\mathrm{mCerbDO}_{2}\right)$ was calculated from the formula: $\mathrm{mCerbDO}_{2}=(\mathrm{CBF} \times((1.39 \times \mathrm{Hb} \times \mathrm{Hbsat} / 100)+(0.00$ $\left.3 \times \mathrm{PaO}_{2}\right)$ ); where total internal carotid blood flow was used as a surrogate of $\mathrm{CBF} ; \mathrm{Hb}=$ Hemoglobin concentration $(\mathrm{g} / \mathrm{dl}) ; \mathrm{Hbsat}=$ Hemoglobin saturation. Modified cerebral oxygen consumption $\left(\mathrm{mCerbVO}_{2}\right)$ was calculated according to the Fick principle, i.e., $\mathrm{mCerbVO}_{2}=\left(\mathrm{CBF} \times\left(\mathrm{SaO}_{2}-\mathrm{TOI}\right)\right)$ where TICF was used as a surrogate of $\mathrm{CBF}$ and TOI was used as a surrogate of cerebral venous oxygen saturation (15).

\section{Statistical Analysis}

Neonatal demographic data were compared using ANOVA. Frequency data were analyzed using Fishers exact test. Differences in cerebral hemodynamic and oxygenation measures according to $\mathrm{MgSO}_{4}$ exposure were assessed using repeated measures ANOVA with post-hoc analysis by paired $t$-test comparisons using the Bonferroni correction. Regression analyses were conducted to assess the associations between total magnesium sulfate dose and all hemodynamic variables, including gestational age and hemoglobin in the regression models. Statistical analyses were performed with SPSS v20.0 (SPSS, Chicago, IL) with a $P$ value $<0.05$ considered significant.

\section{STATEMENT OF FINANCIAL SUPPORT}

Financial support for this study was provided by a Channel 7 Research Foundation Project Grant, Australia and a Women's and Children's Hospital Research Foundation Project Grant, Adelaide, Australia.

Disclosure: The authors have no potential /perceived conflicts of interest to disclose. 


\section{REFERENCES}

1. Fanaroff AA, Stoll BJ, Wright LL, et al.; NICHD Neonatal Research Network. Trends in neonatal morbidity and mortality for very low birthweight infants. Am J Obstet Gynecol 2007;196:147.e1-8.

2. Kuban KC, Leviton A, Pagano M, Fenton T, Strassfeld R, Wolff M. Maternal toxemia is associated with reduced incidence of germinal matrix hemorrhage in premature babies. J Child Neurol 1992;7:70-6.

3. American College of O, Gynecologists Committee on Obstetric P, Society for Maternal-Fetal M. Committee Opinion No. 455: Magnesium sulfate before anticipated preterm birth for neuroprotection. Obstet Gynecol 2010;115:669-71.

4. Petrova A, Mehta R. Magnesium sulfate tocolysis and intraventricular hemorrhage in very preterm infants. Indian J Pediatr 2012;79:43-7.

5. Doyle LW, Crowther CA, Middleton P, Marret S, Rouse D. Magnesium sulphate for women at risk of preterm birth for neuroprotection of the fetus. Cochrane Database Syst Rev 2009:CD004661.

6. Rantone TH, Grönlund JU, Jalonen JO, et al. Comparison of the effects of antenatal magnesium sulphate and ritodrine exposure on circulatory adaptation in preterm infants. Clin Physiol Funct Imaging 2002;22:13-7.

7. Antonov SM, Johnson JW. Permeant ion regulation of N-methyl-Daspartate receptor channel block by $\mathrm{Mg}(2+)$. Proc Natl Acad Sci U S A 1999;96:14571-6.

8. Suzuki-Kakisaka H, Sugimoto J, Tetarbe M, Romani AM, Ramirez Kitchen CM, Bernstein HB. Magnesium sulfate increases intracellular magnesium reducing inflammatory cytokine release in neonates. Am J Reprod Immunol 2013;70:213-20.

9. Imamoglu EY, Gursoy T, Karatekin G, Ovali F. Effects of antenatal magnesium sulfate treatment on cerebral blood flow velocities in preterm neonates. J Perinatol 2014;34:192-6.

10. Belfort MA, Saade GR, Moise KJ Jr. The effect of magnesium sulfate on maternal and fetal blood flow in pregnancy-induced hypertension. Acta Obstet Gynecol Scand 1993;72:526-30.

11. Shokry M, Elsedfy GO, Bassiouny MM, Anmin M, Abozid H. Effects of antenatal magnesium sulfate therapy on cerebral and systemic hemodynamics in preterm newborns. Acta Obstet Gynecol Scand 2010;89:801-6.

12. Paradisis M, Osborn DA, Evans N, Kluckow M. Randomized controlled trial of magnesium sulfate in women at risk of preterm delivery-neonatal cardiovascular effects. J Perinatol 2012;32:665-70.

13. Greisen G, Børch K. White matter injury in the preterm neonate: the role of perfusion. Dev Neurosci 2001;23:209-12.

14. Altman DI, Perlman JM, Volpe JJ, Powers WJ. Cerebral oxygen metabolism in newborns. Pediatrics 1993;92:99-104.

15. Balegar KK, Stark MJ, Briggs N, Andersen CC. Early cerebral oxygen extraction and the risk of death or sonographic brain injury in very preterm infants. J Pediatr 2014;164:475-80.e1.

16. Noori S, McCoy M, Anderson MP, Ramji F, Seri I. Changes in cardiac function and cerebral blood flow in relation to peri/intraventricular hemorrhage in extremely preterm infants. J Pediatr 2014;164:264-70.e1-3.

17. Ayromlooi J, Desiderio DM, Tobias M, Berg P. Effect of magnesium sulfate on maternal and fetal hemodynamics and fetal brain function and metabolism. Pediatr Pharmacol (New York) 1982;2:305-15.

18. Verhagen EA, Kooi EM, van den Berg PP, Bos AF. Maternal antihypertensive drugs may influence cerebral oxygen extraction in preterm infants during the first days after birth. J Matern Fetal Neonatal Med 2013;26: 871-6.

19. Pezzati M, Giani T, Gambi B, et al. Influence of maternal magnesium sulphate and ritodrine treatment on cerebral blood flow velocity of the preterm newborn. Acta Obstet Gynecol Scand 2001;80:818-23.
20. Mittendorf R, Dammann O, Lee KS. Brain lesions in newborns exposed to high-dose magnesium sulfate during preterm labor. J Perinatol 2006;26:5763.

21. Euser AG, Cipolla MJ. Magnesium sulfate for the treatment of eclampsia: a brief review. Stroke 2009;40:1169-75.

22. Fairlie FM. Doppler flow velocimetry in hypertension in pregnancy. Clin Perinatol 1991;18:749-78.

23. Rasmussen K. Fetal haemodynamics before and after treatment of maternal hypertension in pregnancy. Dan Med Bull 1987;34:170-2.

24. Stark MJ, Clifton VL, Wright IM. Neonates born to mothers with preeclampsia exhibit sex-specific alterations in microvascular function. Pediatr Res 2009;65:292-5.

25. Hallak M, Berry SM, Madincea F, Romero R, Evans MI, Cotton DB. Fetal serum and amniotic fluid magnesium concentrations with maternal treatment. Obstet Gynecol 1993;81:185-8.

26. Hallak M, Cotton DB. Transfer of maternally administered magnesium sulfate into the fetal compartment of the rat: assessment of amniotic fluid, blood, and brain concentrations. Am J Obstet Gynecol 1993;169(2 Pt 1):427-31.

27. McDonald JW, Silverstein FS, Johnston MV. Magnesium reduces $\mathrm{N}$-methyl-D-aspartate (NMDA)-mediated brain injury in perinatal rats. Neurosci Lett 1990;109:234-8.

28. Espinoza MI, Parer JT. Mechanisms of asphyxial brain damage, and possible pharmacologic interventions, in the fetus. Am J Obstet Gynecol 1991;164(6 Pt 1):1582-9; discussion 1589-91.

29. de Haan HH, Gunn AJ, Williams CE, Heymann MA, Gluckman PD. Magnesium sulfate therapy during asphyxia in near-term fetal lambs does not compromise the fetus but does not reduce cerebral injury. Am J Obstet Gynecol 1997;176(1 Pt 1):18-27.

30. Andersen CC, Pillow JJ, Gill AW, et al. The cerebral critical oxygen threshold of ventilated preterm lambs and the influence of antenatal inflammation. J Appl Physiol (1985) 2011;111:775-81.

31. Doyle LW, Anderson PJ, Haslam R, Lee KJ, Crowther C; Australasian Collaborative Trial of Magnesium Sulphate (ACTOMgSO4) Study Group. School-age outcomes of very preterm infants after antenatal treatment with magnesium sulfate vs placebo. JAMA 2014;312:1105-13.

32. Wijbenga RG, Lemmers PM, van Bel F. Cerebral oxygenation during the first days of life in preterm and term neonates: differences between different brain regions. Pediatr Res 2011;70:389-94.

33. Grant PE, Roche-Labarbe N, Surova A, et al. Increased cerebral blood volume and oxygen consumption in neonatal brain injury. J Cereb Blood Flow Metab 2009;29:1704-13.

34. SA Maternal \& Neonatal Clinical Network. Magnesium sulphate for neuroprotection of the fetus in women at risk of preterm birth. South Australian Perinatal Practice Guidelines. South Australia: Department of Health, Government of South Australia, 2012.

35. Papile LA, Burstein J, Burstein R, Koffler H. Incidence and evolution of subependymal and intraventricular hemorrhage: a study of infants with birth weights less than 1,500 gm. J Pediatr 1978;92:529-34.

36. Skov L, Ryding J, Pryds O, Greisen G. Changes in cerebral oxygenation and cerebral blood volume during endotracheal suctioning in ventilated neonates. Acta Paediatr 1992;81:389-93.

37. Van Bel F, Van de Bor M, Stijnen T, Baan J, Ruys JH. Cerebral blood flow velocity changes in preterm infants after a single dose of indomethacin: duration of its effect. Pediatrics 1989;84:802-7.

38. Hoecker C, Nelle M, Poeschl J, Beedgen B, Linderkamp O. Caffeine impairs cerebral and intestinal blood flow velocity in preterm infants. Pediatrics 2002;109:784-7. 